\title{
Approche communicationnelle et organisationnelle des enjeux du Community Management
}

Communication and organization approaches to the issues of Community

Management

Olivier Galibert

\section{(2) OpenEdition}

\section{Journals}

Édition électronique

URL : http://journals.openedition.org/communicationorganisation/4814

DOI : 10.4000/communicationorganisation. 4814

ISBN : 979-10-300-0155-6

ISSN : 1775-3546

Éditeur

Presses universitaires de Bordeaux

Édition imprimée

Date de publication : 1 décembre 2014

Pagination : 265-278

ISBN : 978-2-86781-905-6

ISSN : $1168-5549$

Référence électronique

Olivier Galibert, « Approche communicationnelle et organisationnelle des enjeux du Community Management », Communication et organisation [En ligne], 46 | 2014, mis en ligne le 01 décembre 2017 , consulté le 10 décembre 2020. URL : http://journals.openedition.org/communicationorganisation/ 4814 ; DOI : https://doi.org/10.4000/communicationorganisation.4814 


\title{
Approche communicationnelle et organisationnelle des enjeux du Community Management
}

\author{
Olivier Golibert ${ }^{1}$
}

\section{Introduction}

Le texte que nous présentons ici marque l'actualisation et la mise en perspective des travaux scientifiques que nous avons menés depuis quinze ans. Il propose un programme de recherche et, de ce fait, retracera les grandes lignes d'une problématisation large qui, à ce stade, ouvre plus de portes qu'elle n'en ferme. L'exercice peut sembler périlleux dans le cadre contraint et limité de l'article scientifique. Il se justifie néanmoins par la singularité de l'étude d'un phénomène communicationnel majeur : la socialisation électronique de nature communautaire, mais également, en creux, par la valeur testimoniale inhérente à la mise en récit d'un parcours scientifique ancré en Sciences de l'Information et de la Communication.

Nous défendrons ici la thèse d'un engagement collectif en ligne indissociablement lié à l'instrumentalisation marketing d'Internet, dont la rationalisation du social induite doit être questionnée. Pour ce faire, nous aborderons la prégnance utilitariste du contexte de diffusion du Community Management qui en découle (Chéreau, 2010 ; Ertzscheid et al., 2010 ; etc.). Cette prégnance utilitariste est accentuée par l'intégration de plus en plus manifeste des dispositifs de communications partagées en ligne dans des politiques d'explicitation et de gestion collaborative des connaissances (Cohendet et al., 2006). Cette double prégnance utilitariste questionne plus que jamais, dans le contexte de la société numérique, l'incompressibilité du monde vécu chère à Habermas (Habermas, 1987), les luttes émancipatoires pour la reconnaissance (Honneth et al., 2013), tout comme la société généreuse, conviviale et réciproque qu'Alain Caillé et les penseurs du MAUSS (Caillé, 2010) appellent de leurs vœux.

Ainsi l'objet de recherche que nous observons demeure la compréhension des formes et des normes de la rationalisation du lien communautaire en

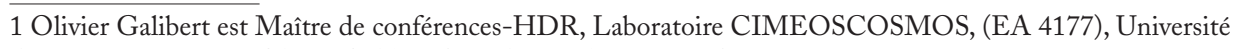
de Bourgogne. Dijon. Olivier.Galibert@iut-dijon.u-bourgogne.fr 
ligne. Au rythme de la massification des usages collaboratifs, coopératifs ou participatifs de l'Internet et des Intranets, cet objet de recherche a évolué pour questionner plus précisément, aujourd'hui, les enjeux du Community Management. Nous émettons l'hypothèse générale que ce Community Management se propage à toutes les sphères de l'activité humaine et qu'il est amené à jouer un rôle de premier choix dans les médiations du savoir à venir.

Afin d'étayer cette proposition, nous reviendrons tout d'abord sur l'instrumentalisation d'origine marketing considérée comme élément fondamental de l'étude et de la compréhension du lien communautaire en ligne. Puis nous nous questionnerons sur la généralisation de cette instrumentalisation en étudiant la construction et la propagation du Community Management. Ensuite, nous proposerons une approche info-communicationnelle théorique et méthodologique du processus d'institutionnalisation du Community Management. Nous envisagerons, alors, l'intérêt d'éclairer notre problématisation à la lumière des phénomènes et processus de médiation des savoirs. Enfin, nous formulerons les grandes ligne d'un programme de recherche se donnant pour objet l'étude d'un Community Management considéré comme outil de gestion (Chiapello \& Gilbert, 2013) mis au service de cette médiation des savoirs.

\section{Pertinence et actualité de l'étude du lien communautaire en ligne et de sa normalisation instrumentale}

Y a-t-il encore aujourd'hui une spécificité de la socialisation électronique, par rapport aux autres dimensions de notre vivre ensemble ? Nos interactions s'appuient désormais, pour tout ou partie, sur le développement massif des TIC. Problématique largement abordée par le sens commun, la séparation entre les interactions en ligne et hors ligne devient difficile à appréhender, voire quasiartificielle. En effet, comment savoir si l'appartenance développée et éprouvée pour un groupe d'amis est due au fait de passer un moment de convivialité, ou parce que les protagonistes échangent intensément sur leurs pages Facebook respectives avant et après le dit événement ? Est-ce que l'appel téléphonique reçu sur un mobile s'inscrit davantage dans un processus de sociabilité que le SMS envoyé préalablement ou le tweet à peine lu ? Sans éluder la question proprement médiologique de l'importance du support, ni les recherches menées sur la spécificité fonctionnelle de telle ou telle technologie, force est de constater que les TIC, peu ou prou et quelles qu'elles soient, participent pleinement du lien grégaire qui lie les individus. Il devient donc délicat de dénouer le fil des processus socialisants artefactuellement supportés de ceux qui ne le sont pas. De ce point de vue, la communauté en ligne n'est pas moins valable, pas moins illusoire, pas moins « incertaine » que la communauté hors ligne. Sans préfigurer ce qui est communautaire de ce qui ne l'est pas dans notre manière d'aborder les formes de socialisation à l'œuvre sur Internet, l'intégration de la reliance électronique comme processus de socialisation 
banal pourrait rendre moins originale la particularité communicationnelle du lien communautaire en ligne par rapport aux modes de socialisation ou de sociabilité « traditionnels », médiatés ou non.

Néanmoins, l'étude de la socialisation électronique occupe, à l'heure du Web dit Social, une bonne partie des recherches en SIC. Et nous partageons cet intérêt. En effet, la compréhension de la socialisation électronique primaire (c'est-à-dire communautaire) constitue le programme de recherche que nous suivons depuis 1995. L'originalité de notre approche infocommunicationnelle réside alors dans la prise en compte systématique de l'impact de la rationalisation potentiellement induite par les stratégies de communication marketing sur les formes " on line " de communalisation (Weber, 1995). Cette perspective semble peu traitée dans notre discipline et, exception faites des Sciences de Gestion elles-mêmes qui l'abordent de manière fonctionnaliste (Amstrong \& Hagel, 1997), peu traitée globalement dans les Sciences Humaines et Sociales. Pourtant, l'appréhension des conséquences de la communication marketing sur la normalisation des SACI (Services et Applications à vocation Communautaire sur Internet) nous apparaît comme l'une des clés de compréhension des usages des technologies Internet aujourd'hui.

C'est ainsi qu'au-delà et en-deçà de l'étude des processus de construction du lien communautaire à l'œuvre, nous nous sommes focalisé sur l'impact de l'instrumentalisation marketing des formes de socialisation primaire médiatée. Cette instrumentalisation peut être observée dès le milieu des années 1990, avec l'apparition des premiers acteurs du e-business aux États-Unis. Le business model d'Internet, basé sur la gratuité et le financement par la publicité, conditionne alors l'attrait pour des sites web censés maintenir « captifs » les Internautes; ceci dans le but de les exposer à des messages publicitaires de plus en plus ciblés. Les forums de discussions ou les chats, formes originelles des SACI, ont rapidement été identifiés comme autant de réponses efficaces à cette " captivité » souhaitée. En effet, les temps de connexion aux sites dits " communautaires ", c'est-à-dire abritant un ou plusieurs SACI, étaient plus longs que la moyenne de temps passé sur un site web classique. Les SACI sont donc devenus la cible des publicitaires qui ont commencé, dès cette période, à développer un arsenal de techniques plus ou moins personnalisées et invasives afin de toucher leurs publics et donner « de la valeur au lien »(Cova, 1995). Très rapidement, les marketers comprirent l'intérêt de construire leurs propres SACI, afin d'être en mesure d'attirer plus sûrement des prospects ou de fidéliser des groupes d'internautes autour d'une marque ou d'un produit. L'ensemble des initiatives communautaires éparses menées par les acteurs économiques et industriels d'Internet a, peu à peu, constitué une stratégie d'instrumentalisation du lien communautaire assez cohérente.

Il nous est alors apparu riche de potentialité de questionner la socialisation électronique en interrogeant les effets rationalisant de cette marchandisation 
du lien communautaire. Cette observation longitudinale, initiée lors de nos travaux doctoraux (Galibert, 2003), n'a jamais cessé depuis. Tout en constatant alors la diversification et la généralisation des politiques d'instrumentalisation communautaire, nous observions une forme de survivance d'un idéal de la communauté virtuelle empiriquement basé sur des pratiques communicationnelles soucieuses de garantir des discussions raisonnables et raisonnées (éthique de la discussion), ainsi que des interactions marquées par la réciprocité (logique de don). C'est donc dans le cadre d'une dialectique «émancipation-réification » que nous observions, dès le début des années 2000, les manifestations électroniques du lien communautaire. Avec la massification des usages collaboratifs et participatifs d'Internet, que d'aucuns qualifient un peu hâtivement d'avènement d'un Web 2.0 ( $\mathrm{cf}$ Bouquillion \& Matthews, 2010), nous avons pu constater, vers la fin de la dernière décennie, que l'instrumentalisation généralisée du lien communautaire s'institutionnalisait dans une discipline à part entière de la gestion : le Community Management. Dès lors, comment ce Community management s'articule-t-il au modèle idéal de la communauté virtuelle dans le cadre de la dialectique « émancipationréification "?

\section{Construction et propagation des formes et des normes du Community Management}

$\mathrm{Au}$-delà de la marchandisation du lien communautaire qui en fixe les règles stratégiques et opérationnelles, le vocable «Community Management » prend forme et sens en même temps que se propage l'instrumentalisation des SACI à toutes les activités humaines en ligne. Nous avions déjà observé la nécessité de penser l'instrumentalisation communautaire en ligne en-deçà et au-delà de la sphère marchande dans nos travaux doctoraux entre 1997 et 2003. En effet, les premières formes d'instrumentalisation marketing s'appuyaient alors sur des SACI «à buts non-lucratifs », notamment les forums "USENET », de nature libertaire, très souvent critiques à l'égard de la marchandisation du Web (on se souvient particulièrement de la critique des SPAMS et autres publipostages non désirés, de la critique de l'autopromotion dans les forums, ou de la modération par transfert des posts commerciaux dans des forums "poubelles à prospectus»). De même, les prémices del'Internet militant, étudiés par Fabien Granjon dans les années 1990 (Granjon, 2001), fournissaient une opportunité d'organisation, de mobilisation et de recrutement de nouveaux membres pour les parties-prenantes du tiers secteur. Il est vrai que le rôle modélisateur qu'ont pu représenter les SACI associatifs dans l'élaboration des stratégies de Community Management demeure à interroger. Néanmoins, l'utilisation militante d'outils communautaires préfigure ou se combine à l'instrumentalisation plus sophistiquée des SACI d'origine marketing. Pour reprendre la terminologie wébérienne, nous parlerions alors de propagation 
du Community Management à la sphère de l'action rationnelle en valeur (Weber, 1995).

La propagation de la figure du Community Management au-delà des politiques marketing se fait sur plusieurs niveaux et en plusieurs étapes. En effet, nous observons tout d'abord la poursuite de la diffusion du Community Management dans les organisations lucratives, d'une part via l'élargissement de l'instrumentalisation marketing aux politiques d'innovation collective (Crowdsourcing) et, d'autre part, via les stratégies 2.0 de communication interne qui convergent avec les courants managériaux récents (management par objectifs, autonomie des collaborateurs, constitution d'équipes projets, démarches qualités, politique de gestion des connaissances et organisation apprenante, insertion des TIC et changement organisationnel, etc.). Ensuite, le développement des réseaux sociaux grand-public et la massification du Community Management marketing induit poussent toutes les organisations à construire les modalités de leur présence en ligne. Pour les professionnels de la communication stratégique, force est de constater qu'il vaut mieux bâtir son identité numérique que de laisser le champ libre à des tiers pas forcément bien intentionnés. L'avènement du Web 2.0 ou Web social agit donc, sous la pression de la massification des usages et des discours d'accompagnement (O'Reilly, 2005), comme une injonction faite à toutes les organisations et les institutions de se doter d'une stratégie de communication 2.0. Cette stratégie utilise le plus souvent une page Facebook ou Google+, liées au site web institutionnel, qui autorise l'interaction avec le Community Manager ou les autres usagers par des actions simples («liker» sur Facebook par exemple) ou des commentaires plus ou moins rédigés. La page Facebook d'une organisation ou d'une institution se présente à la fois comme un miroir se substituant peu à peu au site web classique, mais également comme l'opportunité multimodale d'agréger des fonctionnalités communautaires jusque-là supportées par des dispositifs spécifiques et complexes à développer (ex : sites de Chats, forums de discussions, blogs, etc.).

Engoncés dans ce dictat du 2.0, les administrations, les associations non-lucratives, les établissements publics se dotent de pages Facebook qui nécessitent un suivi et une animation que seul un Community Manager semble être en mesure d'assurer. Ainsi, la grande majorité des organisations et des institutions s'emparent des préceptes et des problématiques stratégiques et opérationnelles du Community Management. Elles le font dans le but de gérer au mieux leur image externe, la relation avec leurs usagers ou adhérents, leur culture organisationnelle ou leur coordination de l'action.

\section{Identifier, analyser et comprendre les enjeux de l'institutionnalisation du Community Management}

Les spécificités culturelles d'Internet, diluées au fil du temps, continuentelles de travailler durablement la manière dont nous interagissons en ligne, 
dont nous construisons nos relations? Le lien communautaire et son modèle idéal et émancipatoire de la communauté virtuelle est-il toujours l'un des fondements normatif à l'œuvre ? Qu'en est-il à l'heure où tous les spécialistes et professionnels de la valeur de lien n'ont qu'un mot à l'esprit : "Community Management"? Ce Community Management cristalliserait toutes les formes de rationalisation à des fins marchandes et/ou managériales mises en place dans les stratégies de communication en ligne pour valoriser le lien entre internautes/intranautes. C'est ce Community Management, considéré comme le cahier des charges de l'animation efficace et de la stimulation des interactions dans un SACI, qu'il soit interne ou externe à l'organisation, qui se présente aujourd'hui comme l'un des facteurs structurants de la socialisation électronique ; et ce Community Management de s’immiscer dans toutes les stratégies de communications numériques, de finir d'imposer la communication stratégique au cœur de toutes les sphères d'activités. En un sens, cette propagation parachève l'actualisation du modèle des relations publiques généralisées proposé par Bernard Miège qui montrait, dès la fin des années 80, l'obligation pour les organisations, qu'elles se donnent un but lucratif ou non, de contrôler leur communication dans le cadre d'une stratégie externe ou interne (Miège, 1989).

Dans ce contexte rationalisé, en quoi la socialisation électronique dans les SACI peut-elle encore préserver une part du monde vécu, cher à Habermas ? Comment des interactions dégagées de l'emprise de l'agir stratégique pourraient-elles se développer ? Est-ce que les modes de valorisation de la parole des usagers-membres des SACI, fixés par les préceptes du Community Management, supportent-ils la présence en ligne d'actes généreux et réciproques ? Plus que jamais, le poids de l'instrumentalisation du lien communautaire en ligne apparaît prégnant quant à la construction des collectifs sur Internet. Malgré tout, l'étude de l'émergence de la forme institutionnalisée de cette instrumentalisation, le Community Management, ainsi que son impact rationalisant, doivent être encore interrogés, selon nous, à la lumière de la dialectique " émancipation/réification ». Car si l'hypothèse de la rationalisation du social par le Community Management semble plus plausible, il n'en demeure pas moins heuristique de chercher de manière systématique les traces éventuelles du modèle émancipatoire de la communauté virtuelle et de sa possible reconfiguration. Et cette recherche débute par l'examen de l'articulation sémantique et symbolique entre les catégories de «communauté » et de « management».

La notion de Community Management associe donc les termes antinomiques de « communauté » et de " management ». L'un se référant à une socialisation primaire (Caillé, 2000) et l'autre à une socialisation secondaire (Weber, 1995). Cette notion fait ainsi écho aux mutations complexes de l'Espace Public (EP) dans lequel s'inscrit notre questionnement : en effet, si nous émettons l'hypothèse d'une colonisation de l'EP par la communication marketing et 
managériale au travers de l'émergence et de la généralisation des politiques de Community Management, nous formulons concomitamment la contrehypothèse d'une incompressibilité du monde vécu due à la nature même du lien communautaire en ligne et de ses conditions d'émergence. Contre toute attente, le Community Management, s'il peut apparaître comme un facteur de rationalisation, doit également être envisagé au travers des résistances qu'il suscite, voire des limites qu'il intègre lui-même à son pouvoir rationalisant.

Afin d'étayer cette proposition, nous nous sommes notamment donné pour objectif d'observer la manière dont les Community Managers intégraient la nature limitée de leurs actions, faisant de cette autolimitation l'une des caractéristiques intrinsèques de ce soft-management du social en ligne. Cette perspective nous a amené à poser les bases empiriques et méthodologiques de l'étude communicationnelle de la figure du Community Management. D'un point de vue général, nous la menons à travers un programme empirique qui se donne notamment pour corpus les traces institutionnalisées et institutionnalisantes qui le constituent. Il s'agit des traces régulatrices de nature juridiques (ex : les chartes communautaires, les explicitations de politiques de modérations aux usagers, etc.), des traces expertes (ex : l'étude de la littérature spécialisée, l'analyse des sites de Web agencies spécialisées en Community Management), des traces de la professionnalisation des "Community Managers » (ex : fiches métiers, fiches compétences, contenus pédagogiques de formations certifiantes et diplômantes dédiées). Cette étude des traces se voit complétée par l'analyse des pratiques professionnelles et des représentations « métiers » des acteurs du Community Management (ex : étude de témoignages de Community Managers dans les médias et les sites web spécialisés, étude nethnographique de communautés de pratiques dédiées aux Community Managers).

\section{Enjeux des SACI comme dispositifs communicationnels de médiation des savoirs}

$\mathrm{Au}$ fil de nos recherches et de nos terrains d'observation passés et en cours (Galibert, 2014), l'institutionnalisation de la rationalisation de l'action communautaire en ligne nous semble parfaitement visible. S'inscrivant dans le processus de généralisation de l'instrumentalisation marketing et managérial des SACI, cette institutionnalisation s'observe avec intérêt aujourd'hui dans le champ de la médiation du savoir. Si la vocation initiale des « communautés virtuelles » chères à Howard Rheingold (Rheingold, 2001) peut paraître liée à la construction de nouvelles solidarités organiques, elle ne peut s'envisager sans l'intérêt commun qui prévaut très souvent à sa création : l'accès à une information discutée, vérifiée, validée et actualisée. En ce sens, si les SACI peuvent parfois devenir des dispositifs sociotechniques de socialisation sans visée utilitariste, ils n'en demeurent pas moins des supports collaboratifs, coopératifs et participatifs d'échange, de partage 
et de diffusion de savoirs. Les internautes «visitent» un SACI pour les informations proposées par les usagers-membres et/ou disponibles dans la mémoire collective de la communauté. Ils y reviennent pour les connaissances en cours de constitution auxquelles ils contribuent par des questions, des commentaires, des remerciements, des encouragements, voire des invectives. Ainsi, pour revendiquer un certain potentiel reliant, les SACI doivent avant tout prouver leur efficacité en tant que dispositifs de production, diffusion et fixation des savoirs. Intégrer un SACI donne accès aux traces écrites plus ou moins organisées de la mémoire collective (par exemple les discussions de forums de discussion, voire la rubrique des Foires Aux Questions), ainsi qu'aux personnes ressources elles-mêmes.

Dans ce cadre, au-delà d'une approche diffusionniste de l'information, les SACI peuvent être considérés comme des dispositifs d'explicitation et de partage de connaissances. Mais peuvent-ils s'inscrire dans une « [...] véritable perspective communicationnelle fondée sur la co-construction de sens, sur le débat et sur l'altérité » (Bonnet et al., 2010) ? L'accès aux savoirs partagés, explicités et formalisés par des profanes ou des amateurs en ligne (Flichy, 2010) participe-t-il systématiquement de la résurgence émancipatoire des SACI présente dans le modèle de la communauté virtuelle ? Comme nous venons de le proposer, l'usage d'un SACI serait ainsi principalement motivé par l'accès à un dispositif de partage de connaissances et d'expériences. Si cette hypothèse venait à être validée, elle pourrait ramener l'ensemble des usagers des SACI dans une communication à visée utilitaire. Il s'agirait alors pour ces derniers de profiter de l'information et des ressources collectives disponibles dans une logique consumériste (cf. figure du lecteur passif dit "lurker ») ou d'interagir avec les autres usagers-membres uniquement dans l'idée d'une accumulation et d'une valorisation de son capital informationnel. Ce basculement vers la dimension rationalisante des SACI se trouverait alors accentuée par l'action des community managers, que l'on doit d'ores et déjà considérer comme des professionnels de la médiation des savoirs.

Ainsi, il nous apparaît aujourd'hui primordial d'étudier les normes et les formes organisationnelles de la socialisation en ligne comme autant de dispositifs de médiation des savoirs ; savoirs instrumentalisés massivement par le marketing et le management, participant d'activités inscrites dans des logiques marchandes ou non-marchandes, lucratives ou non lucratives, notamment dans des contextes de " communication d'action et d'utilité sociale »(Bernard, 2008). Les SACI se présentent comme des espaces de communication dans lesquels des savoirs sont en cours de création collective, disponibles et mobilisables. Au-delà d'une vision substantialiste qui les définirait comme de simples bases de données auto-spontanées, ils doivent s'étudier dynamiquement comme autant de traces d'actualisation de connaissances et d'intelligence collective. Dans une perspective plus critique, nous devons les appréhender en lien avec les débats sur la société 
de l'information et de la connaissance. En effet, les savoirs accessibles via les SACI représentent un enjeu stratégique pour un certain nombre d'acteurs. Il s'agit notamment des membres du SACI eux-mêmes qui ont intérêt à ce que l'activité communicationnelle soit la plus dense possible pour disposer d'un maximum de contenus et accéder à des points de vues opposés débouchant parfois sur de véritables controverses. C'est également l'intérêt des community builders, propriétaires des sites intégrant ces SACI, qui pourront valoriser, le plus souvent économiquement (sphère marchande) ou politiquement (organisations militantes, collectivités territoriales, institutions, société civile, etc.), l'audience qualifiée que représentent les membres, ainsi que les connaissances produites par ces derniers. Les SACI apparaissent donc, sur le plan de la communication stratégique, comme autant de dispositifs de médiation des savoirs " gagnant-gagnant " qui explicitent, actualisent et diffusent des connaissances, et dont le contenu informationnel auto-généré par les membres bénéficie de fait de la « sagesse de la foule » (Surowiecki, 2008).

\section{Le Community Management au service de la médiation des savoirs ? Un renouvellement problématique et empirique}

Dès lors, si la motivation initiale d'intégration d'un SACI se veut essentiellement " cognitive », subsume-t-elle l'ensemble des phénomènes relationnels qui se jouent dans l'interaction en ligne ? Compte tenu du primat cognitif ou informationnel qui semble se dessiner quant à l'usage des SACI, est-il toujours pertinent de les questionner en tant que dispositifs de socialisation, a fortiori de socialisation primaire ? En effet, à considérer uniquement les SACI comme des bases de données, l'étude de l'accès à l'information pertinente pourrait mettre au second plan, dans le cadre d'un paradigme informationnel centré sur la communication homme-machine, toute approche compréhensive des interactions en ligne.

Là n'est bien sûr pas notre propos, ni notre constat. Car si la visée utilitaire à l'usage d'un SACI ne doit pas être sous-évaluée, elle ne peut, malgré tout, justifier à elle seule la nature des échanges à l'œuvre. En effet, il ne faut pas perdre de vue tout d'abord que les SACI, en tant que dispositifs sociotechniques de médiation des savoirs, possèdent de facto un potentiel de reconnaissance, en préparant par exemple des individus " méprisés » (Honneth, 2008) mais informés à œuvrer pour une critique pratique, voir à co-construire une forme de critique théorique. Comme nous avons pu le dire plus avant, l'accès aux savoirs, qu'ils soient experts et, en ce qui nous concerne, principalement profanes, participe naturellement d'un processus d'émancipation par la connaissance. Ensuite, d'autres dimensions plus « sensibles » sont au cœur de nos préoccupations et de nos investigations. Il nous semble notamment nécessaire de poursuivre l'élaboration et l'autocritique du modèle normatif de la communauté virtuelle à l'aune des dimensions émancipatrices que 
sont le don ou l'éthique de la discussion; dimensions qui, paradoxalement, s'actualisent pour partie dans les principes et les politiques de Community Management. Il est clair pour nous que l'intérêt compréhensif porté à ses dimensions anti-utilitaristes remet la normalisation des relations en ligne au cœur de nos investigations.

Le modèle normatif de la communauté virtuelle se base sur une vision idéalisée de la socialisation communautaire en ligne. Cet idéal de la communauté virtuelle accompagne ou contrebalance la logique utilitariste portée par des politiques de Community Management de plus en plus liées aux enjeux socio-économiques de la médiation des savoirs. L'étude du Community Management, notamment dans le cadre du processus d'institutionnalisation dont il fait l'objet, constitue un objet de recherche communicationnel qui renouvelle l'heuristique critique de la tension "émancipation-réification ». C'est l'étude de cette tension qui constitue le programme scientifique que nous souhaitons poursuivre au coeur des Sciences de l'Information et de la Communication. Pour résumer, ce programme vise à valider ou invalider l'hypothèse générale d'une institutionnalisation du Community Management participant d'une rationalisation du social à l'intérieur des SACI (et donc d'une validation ou d'une invalidation empirique du potentiel normatif du modèle de la communauté virtuelle). Ce programme implique :

1) de problématiser la tension entre le modèle normatif émancipatoire de la communauté virtuelle et la rationalisation du monde vécu colonisé par les enjeux opérationnels et stratégiques d'un Community Management en cours d'institutionnalisation;

2) d'étudier cette tension dans un contexte empirique d'affirmation d'usages communautaires de nature informationnelle et cognitive, semblant mettre au second plan la dimension proprement reliante des dispositifs sociotechniques de communications partagées ;

3) de préparer un étayage empirique de la dialectique " émancipationréification " via l'étude des formes plus ou moins institutionnalisées du Community Management dans les SACI explicitement dédiés à la médiation des savoirs.

\section{Conclusion}

Partant de l'étude de la rationalisation managériale et marketing du lien communautaire en ligne, nous avons développé une approche communicationnelle critique de pratiques spécifiques issues des Sciences de Gestion. Cela nous a conduit à poser un regard distancié, d'une part sur la communication marketing induite par l'instrumentalisation commerciale des dispositifs d'échanges partagés sur Internet et, d'autre part, sur les méthodes d'ingénierie symbolique déployées dans les organisations via les services de communication interne. 
Ensuite, au-delà de ces apports théoriques et méthodologiques, l'évolution de notre objet de recherche nous a amené à interroger les Services et Applications à vocation Communautaire sur Internet et Intranet comme dispositifs infocommunicationnels de médiation des savoirs, dans des contextes lucratifs et non-lucratifs. Ces contextes font et ont déjà fait l'objet d'études empiriques que nous menons ou dirigeons dans des champs aussi différents que la communication et l'éducation environnementale, la communication publique et politique, la santé ou l'e-learning. Cette démarche s'est naturellement accompagnée d'une confrontation au champ des Sciences de l'Education dont les enjeux épistémologiques n'ont malheureusement pu être abordés ici. Il aurait été par exemple éclairant d'entreprendre la reformulation d'une certaine légitimité communicationnelle préalable à l'étude de phénomènes habituellement traités par les didacticiens, les psycho-cogniciens ou les sociologues de l'éducation.

Cependant, malgré les limites explicitées et les effets de cohérence $a$ posteriori inhérents à l'exercice de synthèse auquel nous nous sommes livré ici, nous espérons avoir présenté un programme de recherche propre à mobiliser les énergies pour les années à venir. En effet, il circonscrit une problématisation générale qui nécessitera de nombreux travaux de terrain. L'affinement et le renouvellement des hypothèses que nous formulions dès notre thèse de doctorat constituent, in fine, l'ossature d'un programme de recherche qui n'est pas un programme de rupture, et que nous souhaitons consolider dans un contexte sociétal qui ne cesse d'interroger les causes et les conséquences de la socialisation électronique.

\section{BIBLIOGRAPHIG}

ARMSTRONG A.-H. \& HAGEL J.-H. (1997), Net.gain, expanding markets through virtual communities. Harvard Business School Press. Boston. USA.

BERNARD Françoise (2008), "Recherche-action et communication d'action d'utilités sociétales : le cas de la communication engageante et de l'environnement ", in Bouzon Arlette, Meyer Vincent, Société française des sciences de l'information et de la communication (2008). La communication des organisations entre recherche et action. Paris. L'Harmattan.

BONNET Jacques, BONNET Rosette, RAICHVARG Daniel (2010), La question des savoirs du point de vue des Sciences de l'Information et de la Communication. In Bonnet Jacques, Bonnet Rosette, Raichvarg Daniel (dir.), (2010). Les savoirs communicants: entre histoire, usages et innovations. Dijon. Éditions Universitaires de Dijon.

BOUQUILLION Philippe \& MATTHEWS Jacob Thomas (2010), Le web collaboratif mutations des industries de la culture et de la communication. Grenoble: Presses universitaires de Grenoble.

CAILLÉ Alain (2000), Anthropologie du don : le tiers paradigme, Paris. Ed. Desclée de Brouwer. 
CAILLÉ Alain (2010), «Du convivialisme vu comme un socialisme radicalisé et universalisé (et réciproquement)». In Caillé A., Humbert M., De la convivialité dialogues sur la société conviviale à venir. (2010). Paris : La Découverte

CHÉREAU Mathieu (2010), Community management comment faire des communautés web les meilleures alliées des marques. Paris, Dunod.

CHIAPELLO Ève \& GILBERT Patrick (2013), Sociologie des outils de gestion introduction à l'analyse sociale de l'instrumentation de gestion avec la collaboration de Céline Baud, Marion Brivot, Carine Chemin-Bouzir... [et al.]. Paris : La Découverte.

COHENDET Patrick, CRÉPLET Frédéric, \& DUPOUËT Olivier (2006), La gestion des connaissances firmes et communautés de savoir. Paris. Économica.

COVA Bernard (1995), Au-delà du marché : quand le lien importe plus que le bien. Coll. Dynamiques d'entreprises. Paris : L'Harmattan.

ERTZSCHEID Catherine, FAVERIAL Benoît \& GUÉGUEN Sylvain (2010), Le community management stratégies et bonnes pratiques pour interagir avec vos communautés préface de Lionel Fumado. Paris. Diateino.

FLICHY Patrice (2010), Le sacre de l'amateur. Sociologie des passions ordinaires à l'ère numérique. Paris. Seuil.

GALIBERT Olivier (2003), Les communautés virtuelles. Entre marchandisation, don et éthique de la discussion, Thèse de Doctorat en Sciences de l'Information et de la Communication. Sous la dir. de Bernard Miège l'Université de Grenoble 3. Grenoble.

GALIBERT Olivier (2014), "Vers une instrumentalisation généralisée du lien communautaire en ligne : la montée en puissance du community management ». In Valérie Lépine, Fabien Martin-Juchat et Christelle Fourier (dir.) La communication en débat, des représentations aux pratiques. Grenoble. PUG.

GRANJON Fabien (2001), L'Internet militant mouvement social et usages des réseaux télématiques. Rennes. Éd. Apogée.

HABERMAS Jürgen (1987), Théorie de l'agir communicationnel. Tome 1 Rationalité de l'agir et rationalisation de la société. Paris. Fayard.

HONNETH Axel \& RUSCH Pierre (2013), La lutte pour la reconnaissance traduit de l'allemand par Pierre Rusch. Gallimard. Paris.

HONNETH Axel (2008), La société du mépris. Vers une nouvelle théorie critique. La Découverte. Paris.

MIÈGE Bernard (1989), La Société conquise par la communication. Grenoble: Presses Universitaires de Grenoble.

O'REILLY T. (2005), «What is the Web 2.0 ». url : http://oreilly.com/pub/a/web2/ archive/what-is-web-20.html?page $=5$

SUROWIECKI James (2008), La sagesse des foules (The Wisdom of Crowds, 2004). Paris. Éditions Jean-Claude Lattès.

WEBER Max, CHAVY Jacques, \& DAMPIERRE Éric de (1995), Economie et société trad. de l'allemand par Julien Freund, Pierre Kamnitzer, Pierre Bertrand... [et al.] sous la dir. de Jacques Chavy et d'Eric de Dampierre. Paris. Pocket. 
Résumé : L'article que nous proposons décrit un programme de recherche ancré en Sciences de l'Information et de la Communication. Il vise à donner un éclairage original sur les enjeux du déploiement de la socialisation électronique de nature communautaire. Il généralise l'étude des formes et des normes de la rationalisation du lien communautaire en ligne. Il questionne plus précisément, aujourd'hui, les enjeux du Community Management. Dans le cadre de ce programme scientifique, nous émettons l'hypothèse générale que le Community Management se propage à toutes les sphères de l'activité humaine et qu'il est amené à jouer un rôle de premier choix dans les nouvelles médiations du savoir.

Mots-Clés : communauté virtuelle, instrumentalisation, web 2.0, Community Management, médiation des savoirs

Abstract: This paper describes a research program within Information and Communication Sciences (ISC). This program is based on the understanding of online socialization processes. Particularely, it is focused on rationalization issues for communities forms and rules online. Since the end of the years 2000, this rationalization for online communities is also known as community Management. We formulate the hypothesis that community management policies spread to whole human activities on the web 2.0, and that community management policies rule the new knowledge mediations.

Keywords : virtual communities, web 2.0, Community Management, knowledge mediation and policies. 
